\title{
Desktop Virtualization of Private Cloud for University Library System
}

\author{
Liping Chen \\ Library of Donghua University, 201620, Songjiang, Shanghai, China
}

\begin{abstract}
Focusing on the design and construction of desktop virtualization of university library system, this article discusses the problems in current information construction of university libraries and makes an introduction to cloud computing and the basic concept and systematic structure of desktop virtualization. Based on the VMware VSphere5 software, overall virtual desktop of university library has been realized, which solves the issues lied in which utility private cloud technology in university library system.

Index Terms - desktop virtualization, private cloud, university library, VMware VSphere5
\end{abstract}

\section{Introduction}

Nowadays, there is growing information construction in colleges and universities with the rapid development of information technology. However, the more in-depth information construction develops, the more problems appear, which puzzles many educationists.

In university libraries, each staff works with one PC which is separate equipment and needs administrators to set up to share files and printers with other PC. Besides that, if the staff have to work at home or in other places out of campus, it is almost impossible. Similarly, in the electronic reading room, each $\mathrm{PC}$ needs to be separately maintained the operation system, upgraded its anti-virus software and installed subject application software regularly, and which always takes plenty of time and labor. And problems caused by computer virus, malicious plug-ins and illegal sites increase the difficulty of management. Meanwhile, users' unstandardized operations bring great risk to the stability of their current software or system.

Since personal mobile office equipment has risen in popularity, university teachers and students almost have more than one mobile device such as personal laptop computer, iPhone, Android mobile phone or tablet. In such an environment, it is a growing requirement that the teachers and students check and use reference books rather through internet access to university library anytime and anywhere than by going to library with the computers there.

Above all, if these points are not addressed urgently, the information construction of college and university library will fail to meet teachers' and students' needs. Fortunately, the application of private cloud architecture in library system has yet to be resolved. This paper takes information system of Donghua University library as an example.

\section{Cloud Computing}

Although cloud computing is one of the most popular phenomena to be discussed in academic world, it is still difficult to define accurately and clearly. Many researchers define cloud computing from different aspects[1-3]. In a general way, cloud computing is a state-of-the-art internetbased technology that delivers Infrastructure-, Platform-, and Software as a Service, including IaaS, PaaS and SaaS. And Cloud computing avoids over-provisioning IT infrastructure and training personnel. Since cloud computing has numerous advantages for dairy lives, eg. education, currently, many organizations are building their own private clouds from their existing infrastructure. North Carolina State University developed the Virtual Computing Laboratory which enabled students to reserve and access virtual machines [1]. Moreover, cloud computing is also utilized as a base for modern eLearning applications [4]. Essentially, the bottom layer infrastructure of cloud technology is virtualization technology.

Desktop virtualization is a technology which can host user's all the configuration and application to the server while user just needs one computer terminal accessing the Internet. Because all application can be installed, deployed, and run in the background service, user's desktop is just an image, and all security control is centrally deployed in server, the security has been greatly improved. Besides that, desktop virtualization enables maintenance to be done on the server which greatly reduces maintenance cost.

With the constant development of virtualization technology, a lot of software can be used to build virtualization architecture. Particularly, Ctrix's Xen Server and Xen Desktop, Microsoft's Windows Server2008 Hyper-V, and VMware's VSphere ESXi and View are all virtualization software. During them, due to its friendly man-machine interface and system compatibility, the VMware virtualization software has achieved many users' satisfaction. Therefore, this paper also utilizes the VMware virtualization software to build virtual desktop.

\section{Desktop Virtualization Implementation of Private Cloud Framework for University Library}

The desktop virtualization architecture is based on server virtualization in this paper. The virtual system of private cloud framework is shown in Fig1. 


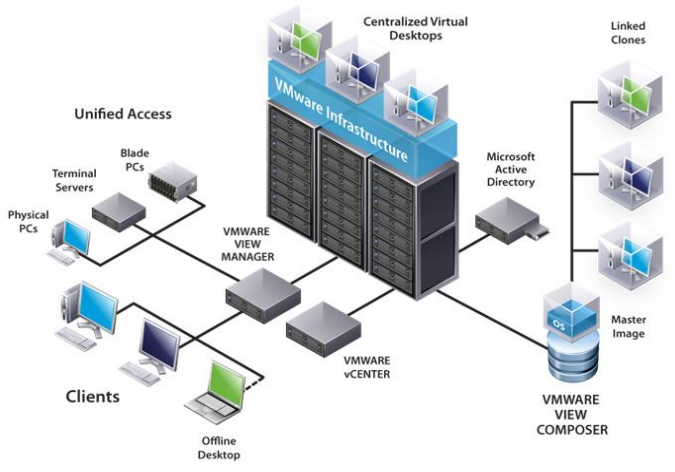

Fig. 1: Virtual System of Private Cloud Framework.

\subsection{Installation of Virtualization Components}

Desktop virtualization system generally consists of View Connection Server, View Security Server, View Agent, View Client, VMware View Composer and Windows Active Directory (Windows AD) Server.

In practice, firstly, a Windows AD server, whose domain is named as view.lib.dhu.edu.cn, is build and then its name is ad.view.lib.dhu.edu.cn. The AD server is used as an authentication server of desktop virtualization. Secondly, set up the View Connect Server and build a domain named view.lib.dhu.edu.cn, which is used for unified management of desktop virtualization platform and similar as vCenter Server of virtual server. Thirdly, Composer components are installed on vCenter server to take snapshots as template of virtual desktop, which can greatly decline the storage consume for deploying virtual desktops. Fourthly, one virtual machine of Window XP or win7 is utilized and configured with common software like Office, player and library's applications. Fifthly, in order to use the virtual machine as module of desktop virtualization, View Agent is set up. Finally, View Secrecy Server is installed as authorization server, when users of external network and untrusted network access to the desktop virtualization network to employ the mobile office environment.

\subsection{Deployment of Virtual Desktop}

Till Windows AD, View Connect Server, Composer, View Secrecy Server and View Agent are installed, virtual desktops are deployed.

A snapshot of the installed Win7 virtual machine is created, and then $\log$ into Connect Server through Web interface to build a new desktop pool. Considering about the difference of demands between library staffs and e-reading room, four desktop pools are built for library staffs in different departments, which are permanent. Once user login, the virtual desktop is bound with the account wherever and whenever he logins unless the administrator changes it manually. It gives users a personalized computing experience that stays with them each time they visit the library, like having their own notebook PCs.

For e-reading room, two extra desktop pools are built, and they're active, that is, to redistribute a desktop for him, each time the user logs in e-reading room site. Furthermore, it wouldn't contain one record for each logged in user which meets the requirement of liquidity of e-reading room.

All the six desktop pools above are automatic deployment pools, that is, if the crashes occur for one desktop by misoperation of users, the administer just needs to $\log$ in the Connect Server to delete the desktop and the system will redeploy new desktop again. All the work are convenient and it just takes 30-40 minutes while deploying 100 desktops. Especially, the rest operations are unattended as long as the deployment command is executed.

As soon as desktop deployment is completed, library staffs and students could log in library and connect to their own virtual desktop. So far cloud architecture of desktop virtualization is fully completed.

Finally, after the completion of architecture, View Connect Server white-lists View Secrecy Server.

\section{Experiment and Result}

Two usability tests were applied: an automatic deployment of desktop test and a multi-terminal connection and safety of desktop test.

\subsection{Automatic Deployment of Desktop}

In order to testing automatic deployment of desktop, a desktop pool is manually created, configured desktop's snapshot ,name, number and property (permanent or active) . After click "ok", check the Connect Server's desktop deployment. Then the deployment completed, the View Client connects to one desktop to check if the desktop is satisfactory. Till confirmation is completed, randomly delete 3 desktops in the pool, then system automatically executes deployment command in 30 seconds and the deleted desktop are redeployed. The result indicates that the automatic deployment function of desktop works well.

\subsection{Multi-terminal Connection and Safety of Desktop}

Install View Client on the low configuration PCs, new PCs, ipad, and Android phones, respectively. And then take experiential tests through library network, campus network, campus WiFi and public network. Details are as follows.

- Hardware, the experiment result is nearly the same between the two configuration PCs when they are connected to the virtual desktop. There's no difference between ipad and Android phones.

- Network, if the bandwidth is more than $512 \mathrm{~kb}$, the operations are smooth. However, the bandwidth is less than $256 \mathrm{~kb}$, latency of operations is obvious even the connection would be interrupted. Fortunately, the application could restore as soon as the reconnection is built.

In addition, the connection to View Client need SSL encryption authentication and account access configuration. Without SSL encryption authentication, the account can't access the desktop system, which ensures the safety of virtualization system. 


\section{Discussion and Conclusion}

Desktop virtualization infrastruction integrates different old computers which scatter throughout the offices and the electronic reading rooms. Through desktop virtualization, old computers are reused and users feel well. Moreover, it takes less than 40 minutes to maintain $120 \mathrm{PCs}$ in the e-reading room by automatically deploying desktop pool, which drastically reduces maintenance time and effort.

The implementation of desktop virtualization makes offcampus mobile office become true. Meanwhile, students can use e-reading sources through virtual desktop wherever they are. In general, it lets more people to experience the convenient of job and learning caused by the cloud environment.

\section{References}

[1] Vouk M, Averritt S, Bugaev M, Kurth A, Peeler A, Schaffer H, Sills E, Stein S, Thompson J (2008) Powered by VCL-using Virtual Computing Laboratory (VCL) Technology to Power Cloud Computing. In: Proceedings of the 2nd international conference on the virtual computing initiative (ICVCI'08)

[2] Foster I, Zhao Y, Raicu I, Lu S (2008) Cloud computing and grid computing 360-degree compared. In: Proceedings of the grid computing environments workshop (GCE'08), Austin, Texas, USA

[3] Armbrust M, Fox A, Griffith R, Joseph AD, Katz R, Konwinski A, Lee G, Patterson D, Rabkin A,Stoica I, Zaharia M (2009) Above the clouds: a Berkeley view of cloud computing. Technical Report UCB/EECS2009-28, University of California at Berkeley

[4] Dong B, Zheng Q, Yang J, Li H, Qiao M (2009) An E-learning ecosystem based on cloud computing infrastructure. In: Ninth IEEE international conference on advanced learning technologies, ICALT 2009, pp 125-127 